\title{
An alternate point-wise scheme for Electric Field Integral Equations
}

\author{
Gabriele Rosati , Juan R. Mosig \\ Laboratory of Electromagnetics and Acoustics (LEMA) \\ Ecole Polytechnique Fédérale de Lausanne (EPFL), CH-1015 Lausanne, Switzerland \\ Received $* * * * * * * * * * * * * * *$; accepted $* * * * * * * * * * * * * *$
}

\begin{abstract}
A point-wise approach that can be used efficiently in the numerical solution of Electric Field Integral Equations is introduced. The algorithm is based on the so-called magic distance concept, which defines exactly the point-to-point equivalent of a four dimensional integral. Magic distance values are rigorously obtained in the electrostatic case and their usefulness is demonstrated. The concept is generalized to the electrodynamic case, resulting in a family of very simple "magic-distance inspired" algorithms that maintain the overall accuracy of Galerkin formulations without appreciable deterioration in the overall accuracy.

(C) 2013 Wiley Periodicals, Inc. Int J RF and Microwave CAE xx:xxx-xxx, 2013
\end{abstract}

Keywords:Electric Field Integral Equation (EFIE), method of moments (MoM), Galerkin, pointmatching

Correspondence to: G. Rosati; email: gabriele.rosati@epfl.ch

DOI .

Published online (wileyonlinelibrary.com) 


\section{INTRODUCTION}

Integral Equation (IE) formulations are one of the most successful techniques for the EM analysis of antennas and scatterers [1]. This is specially true when the involved objects can be reduced to combinations of metallic surfaces and volumes embedded in a 3D homogeneous or multilayered medium [2]. The classical discretization scheme and subsequent numerical implementation of IE formulations is usually performed with the help of the method of moments (MoM). In particular, the IE-MoM implementation known as Galerkin method is endowed with variational properties, which guarantees good convergence properties [3].

The most commonly used IE formulation is the Electric Field Integral Equation (EFIE). On one side it involves only the tangential component of an uniquely defined physical quantity, the electric field. In addition, it can be easily applied to metallic open surfaces that are so ubiquitous in modern technologies. Within the EFIE family, perhaps the most useful version is the Mixed Potential Integral Equation (MPIE). As it is well known [1,4], MPIE exhibits source-observer singularities much milder $\left(1 / R^{\wedge} 1\right)$ than the original EFIE (1/R^3) and allows the use of simpler MoM schemes like pointmatching. But as its name says, MPIE is based on the introduction of auxiliary mathematical quantities, the potentials, whose definition, existence and uniqueness are frequently hard to establish in complex media.

This paper introduces some innovative very simple point-wise MoM schemes aiming at reducing the numerical complexity of the Galerkin method without producing a sizeable deterioration in the accuracy of the results. The developments are based in the introduction of a new concept, the so-called "magic distance". This concept will be introduced in a simplified electrostatic case and then implemented in the frame of EFIE and of its weaker singular version, the MPIE. The implementation remains at a proof-of-concept level using free space Green's functions and a simplified mesh, since the extension to more complex situations should not require any additional qualitative development.

\section{THE INTEGRAL EQUATIONS}

As mentioned in the introduction, we will consider open metallic surfaces embedded in a 3D environment and we will concentrate here in the Electric Field Integral Equation (EFIE). EFIE only involves tangential electric fields, which are always mathematically welldefined quantities, since they have an obvious physical meaning. In electrodynamics, EFIE can be written as $[1,4]$ :

$$
\hat{\mathbf{n}} \times\left[\overline{\overline{\mathbf{G}}}_{\mathbf{E J}}\left(\mathbf{r}, \mathbf{r}^{\prime}\right) \otimes \mathbf{J}_{S}(\mathbf{r})\right]_{\mathbf{r} \in S}=-\hat{\mathbf{n}} \times\left.\mathbf{E}^{i}(\mathbf{r})\right|_{\mathbf{r} \in S}
$$

where $\mathrm{S}$ is the combination of all metallic surfaces, $\mathbf{E}^{\mathbf{i}}$ is the incident excitation field, $\mathbf{J}_{\mathbf{S}}$ is the unknown surface current density and $\overline{\overline{\mathbf{G}}}_{\mathrm{EJ}}$ is the dyadic Green's function, kernel of the integral equation. This Green's function will usually include the effect of the material environment surrounding the metallic sheets. Therefore, the complexity of the dyadic GF goes from the closed-from analytical formulation for free space to quite involved expressions, like the combinations of Sommerfeld integrals and analytical series found in the treatment of planar, cylindrical and spherical layered dielectric media [5]. However, in all cases, the EFIE GF intrinsic source- observer singularity is of $1 / \mathrm{R}^{\wedge} 3$ type, where $R=\left|\mathbf{r}-\mathbf{r}^{\prime}\right|$ is the source-observer distance [1,2].

This is obvious in the well-known analytical form of the dyadic Green's function for free space [1]:

$$
\overline{\overline{\mathbf{G}}}_{\mathbf{E J}}\left(\mathbf{r}, \mathbf{r}^{\prime}\right)=\frac{j \omega \mu}{4 \pi}\left(\overline{\overline{\mathbf{I}}}+\frac{\nabla \nabla}{k^{2}}\right) \frac{\exp (-j k R)}{R}
$$

This means that when discretizing the EFIE with a classical Method of Moments (MoM) procedure, a simple scheme like point-matching cannot be used. This strong singularity also prevents the use of otherwise promising approaches like the point-wise Nyström method, based on numerical quadrature rules $[6,7]$.

This spatial singularity problem can be avoided by solving the EFIE in the spectral domain [1,4]. However, spectral approaches rely heavily in the extensive use of 2D Fourier-transforms and are inefficient for arbitrary or non-canonical geometries. In practice, successful implementations of EFIE (FEKO, WIPL-D) remain in the space domain and resort to the use of subsectional basis and a MoMGalerkin approach.

The Galerkin implementation of the EFIE results then in matrices whose elements are given by double surface (and hence 4D) integrals, where - at least in theory- an $1 / \mathrm{R}^{\wedge} 3$ singularity can be integrated.

A more popular strategy is to transform the EFIE into a mixed potential integral equation (MPIE). Electromagnetic theory allows us to derive the electric field from two potentials in a classical way

$$
\mathbf{E}=-j \omega \mathbf{A}-\nabla V
$$

Using this equivalence, EFIE can be transformed into MPIE:

$$
\hat{\mathbf{n}} \times\left[\begin{array}{l}
j \omega \int_{S} d s^{\prime} \overline{\overline{\mathbf{G}}}_{\mathbf{A}}\left(\mathbf{r}, \mathbf{r}^{\prime}\right) \cdot \mathbf{J}_{s}(\mathbf{r})+ \\
\nabla \int_{S} d s^{\prime} G_{V}\left(\mathbf{r}, \mathbf{r}^{\prime}\right) \cdot \rho_{s}(\mathbf{r})
\end{array}\right]_{\mathbf{r} \in S}=\hat{\mathbf{n}} \times\left.\mathbf{E}^{i}(\mathbf{r})\right|_{\mathbf{r} \in S}(3 \mathrm{a})
$$

where in addition to the unknown surface current density $\mathbf{J}_{\mathbf{S}}$, a new unknown, the surface charge density $\rho_{S}$, also appears.

It is a well-known fact [1], that the singularities in both the scalar Green's Function $G_{V}$ for the scalar potential and the dyadic Green's Function $\overline{\overline{\mathbf{G}}}_{A}$ for the vector potential exhibit only a mild sourceobserver singularity of the $1 / \mathrm{R}$ type that can be absorbed in the surface source integral. Therefore, simple point-matching discretization can be considered with MPIE.

In particular, in free space, the use of the classical Lorentz' gauge [2] results in the following expressions for the potential Green's functions:

$$
\begin{aligned}
& G_{V}\left(\mathbf{r}, \mathbf{r}^{\prime}\right)=\frac{1}{4 \pi \varepsilon} \frac{\exp (-j k R)}{R} \\
& \overline{\overline{\mathbf{G}}}_{A}\left(\mathbf{r}, \mathbf{r}^{\prime}\right)=\frac{\mu}{4 \pi} \frac{\exp (-j k R)}{R}
\end{aligned}
$$


As it has been previously pointed out, the potentials are somewhat artificial quantities that can be defined in several ways and connected through different gauge equations (Coulomb, Lorentz...). This also applies to the associated Green's functions. For instance, it is well known [8] that in a multilayered media the value of the scalar Green's function $G_{V}$ can depend on the orientation of the original dipole source, a strange situation for a scalar potential which is supposed to be created by a point charge. In these cases, it is necessary to add a correction term to the original EFIE expression (3) [5].

The final integral equation of relevance in this paper is the "electrostatic potential integral equation" (EPIE), viewed here as an useful zero-frequency case of the MPIE formulation.

$$
\hat{\mathbf{n}} \times\left[\nabla \int_{S} d s^{\prime} G_{V}\left(\mathbf{r}, \mathbf{r}^{\prime}\right) \rho_{S}(\mathbf{r})\right]_{\mathbf{r} \in S}=-\hat{\mathbf{n}} \times\left.\mathbf{E}^{i}(\mathbf{r})\right|_{\mathbf{r} \in S}
$$

where the scalar Green's function is now simply:

$$
G_{V}\left(\mathbf{r}, \mathbf{r}^{\prime}\right)=\frac{1}{4 \pi \varepsilon R}
$$

In this case, a usual subsequent transformation is to define an "incident" or "external" potential as $\mathbf{E}^{i}(\mathbf{r})=-\nabla V^{i}$ and to integrate the integral equation between a reference point defined as "ground" and a point on the metallic surfaces.

The final result is:

$$
\left[\int_{S} d s^{\prime} G_{V}\left(\mathbf{r}, \mathbf{r}^{\prime}\right) \cdot \rho_{S}(\mathbf{r})\right]_{\mathbf{r} \in S}+\left.V^{i}(\mathbf{r})\right|_{\mathbf{r} \in S}=C
$$

which is the complete formulation of the EPIE. In a simpler version, there is no external potential and the constant $C$ is the known potentials of the metallic objects respect to ground.

The obvious challenge here is to combine the advantages of EFIE and MPIE, i.e. to be able to cast the problem in terms of a physically well defined quantity like the electric field but avoiding the strong singular EFIE integrals. Then, it should be possible to use the pointwise MoM schemes typical of MPIE, instead of the cumbersome Galerkin approaches usually need to cancel these strong singularities. Therefore, it is of great relevance to investigate under which assumptions point-matching or Nyström like strategies can be directly applied to the EFIE. This is the main purpose of this paper.

\section{MOM DISCRETIZATION}

In the standard application of the MoM discretization [D], the unknown quantity is expanded as a linear combination of basis functions $\mathbf{b}_{j}\left(\mathbf{r}^{\prime}\right)$ and the IE is projected, through an internal product on a set of test functions $\mathbf{t}_{i}(\mathbf{r})$. As a result, the integral equation is discretized and replaced by a linear system, where the MoM matrix elements are given by $4 \mathrm{D}$ integrals which can be written as:

$$
z_{i j}=\int_{S_{i}} d s \mathbf{t}_{i}(\mathbf{r}) \int_{S_{j}} d s^{\prime} \mathbf{b}_{j}\left(\mathbf{r}^{\prime}\right) \overline{\overline{\mathbf{G}}}_{\mathbf{E J}}\left(\mathbf{r}, \mathbf{r}^{\prime}\right)
$$

for the EFIE and

$$
\begin{aligned}
& z_{i j}=\int_{S_{i}} d s \mathbf{t}_{i}(\mathbf{r}) \int_{S_{j}} d s^{\prime} \mathbf{b}_{j}\left(\mathbf{r}^{\prime}\right) \overline{\overline{\mathbf{G}}}_{\mathbf{A}}\left(\mathbf{r} \mid \mathbf{r}^{\prime}\right)- \\
& \frac{1}{k_{0}^{2}} \int_{S_{i}} d s\left[\nabla \cdot \mathbf{t}_{i}(\mathbf{r})\right] \int_{S_{j}} d s^{\prime}\left[\nabla^{\prime} \cdot \mathbf{b}_{j}\left(\mathbf{r}^{\prime}\right)\right] G_{V}\left(\mathbf{r} \mid \mathbf{r}^{\prime}\right)
\end{aligned}
$$

for the MPIE.

In the Galerkin version, we simply have: $\mathbf{t}_{j}(\mathbf{r})=\mathbf{b}_{j}(\mathbf{r})$

Obviously, the calculation of 4D integrals is cumbersome and requires a lot of computational time and resources. This is especially the case for the self-terms (the diagonal terms in the MoM matrix) where there is a need to deal with singular and even hypersingular integrals [9].

We propose to replace the $4 \mathrm{D}$ integral required for each entry of the MoM matrix with the computation of a set of point-to-point interactions between an infinitesimal source and some observation points. Source and observation points are arranged in such a configuration that ensures an exact value for the self-term $(i=j)$ and minimizes the error in key near-diagonal terms. Once determined, the points distribution remains fixed in the calculation of every cell-to-cell interaction, thus leading to a very simple and compact implementation. In fact, the proposed approach can be viewed as the development of simple quadrature rules specifically adapted to a given type of singular integrals. Moreover, when properly executed, this strategy should also lead to errors that naturally vanish asymptotically when the source-observer distance increases without bound.

It is expected that an exact representation of the self-term and an accurate approximation of the first near-term(s), which combine to form the most weighing entries in the MoM matrix, will minimize the error in the final unknown quantities, computed through the resolution of the associated linear system.

In this paper, we present different possible configurations for the electrostatic (Sec. IV) and electrodynamic (Sec. V) problems. It is shown that it is possible in both cases to find a point configuration which allows to approximate the computation of the 4D integral with limited error and to compute the unknown quantity (charge or current) with very good accuracy.

Major benefits of our method are the simplicity of implementation, its applicability to problems where potentials cannot be defined, the reduced demand of computational time and resources due to the replacement of 4D integrals with point-to-point calculations.

To develop our strategy we will consider a classical MoM-Galerkin discretization using Rao-Wilton-Glisson (RWG) rooftops as basis functions defined on simple geometrical meshes formed by rectangular cells. Rectangular or square meshes are of interest by themselves. But in addition, it is always possible to apply the results obtained for rectangular or square cells to trapezoidal/triangular meshes by introducing geometrical affine concepts or using homothetic changes of variables, exactly like when defining twodimensional quadratures over non-canonical surfaces.

In order to clarify the above mentioned ideas, we start dealing with the EPIE (electrostatic case). As previously mentioned, this case is of paramount relevance when full-wave (electrodynamic) problems are cast in terms of a mixed-potential integral equation, since then the 
singular part of the MPIE self-terms (which typically must be either extracted or separately treated) is given by the EPIE self-term.

\section{THE ELECTROSTATIC PROBLEM}

The electrostatic potential integral equation (EPIE), given by eqns. 4, doesn't include any differential operator. Therefore it can be discretized with a Galerkin scheme using scalar piecewise constant subsectional basis and test functions, defined as:

$$
b(\mathbf{r})=t(\mathbf{r})= \begin{cases}1 & \mathbf{r} \in S \\ 0 & \text { otherwise }\end{cases}
$$

The value $z_{\text {self }}$ of a generic MoM self-term is then simply given by

$$
z_{\text {self }}=\int_{S} d s \int_{S} d s^{\prime} \frac{1}{\sqrt{\left(x-x^{\prime}\right)^{2}+\left(y-y^{\prime}\right)^{2}}}
$$

where $S$ is a rectangular cell. Amazingly enough, this 4D integral can be fully computed analytically. If $a, b$ are the dimensions of the rectangle $S$ and $c$ its diagonal we get:

$$
\begin{gathered}
z_{\text {self }}=2\left(a^{3}+b^{3}-c^{3}\right) / 3+ \\
2 a^{2} b \ln [(c+b) / a]+2 a b^{2} \ln [(c+a) / b]
\end{gathered}
$$

Now if the calculus mean theorem is applied to the 4D integral (8) we should get

$$
z_{\text {self }}=a^{2} b^{2} \frac{1}{<\sqrt{\left(x-x^{\prime}\right)^{2}+\left(y-y^{\prime}\right)^{2}}>}=a^{2} b^{2} \frac{1}{R_{m}}
$$

In this last expression, $R_{m}$ is an "average" or "mean" distance in the Galerkin sense, obtained by equating eqns. 9 and 10 . It can be viewed as the distance between a point source and a observation point which produces an effect identical to the effect of a distributed source averaged on all its points. Since this distance embodies in a simple unique point-to-point value a complex interaction described by a four dimensional integral, we can worthily call it a "magic distance". For the specific case where $S$ is a unit square, the magic distance takes the interesting value:

$$
R_{m}=[4 \ln (\sqrt{2}+1)-4(\sqrt{2}-1) / 3]^{-1}=0.3363 . .
$$

The magic distance is also defined by the following remarkable property: if two points are randomly selected within a given domain and the distance between them is computed, the magic distance is the harmonic average of these distances.

To test the variability of this magic distance concept, we have also computed its value when $S$ is a circular domain of radius $a$. While it is obviously not a good MoM practice to decompose an arbitrary surface into circular domains, the results obtained for circular cells are of the uppermost theoretical relevance and they can provide support to the applicability of the magic concept distance to other shapes. The inner surface integral in (8) corresponds physically to the potential created by a constant surface charge density in any point $(x, y)$ of the circular domain where it is defined. This is a classical problem in Electrostatics and its solution can be written in terms of complete elliptic integrals [10]. After some algebraic manipulations, the Galerkin self-term (8) is given by a surface integral over the observation domain of some elliptic functions $\mathrm{E}(t)$. More precisely, taking into account the azimuthal symmetry, we get:

$$
z_{\text {self }}=8 \pi a^{3} \int_{0}^{1} \mathrm{E}(t) t d t=16 \pi a^{3} / 3
$$

This is a very interesting result. To compare it with the unit square, we consider a unit surface circle $\left(\pi a^{2}=1\right)$. Then, according to (10) the magic distance for such a circle is:

$R_{m}=16 / 3 \sqrt{\pi}=0.3323$..

a remarkable result when compared with (11) which shows the robustness of the magic distance concept.

The magic distance concept is a theoretical idea but it can be easily generalized to be transformed into a practical tool. In the self case, the inner integral (associated with the source) is replaced with a point charge located in the cell's center. The outer integral could be replaced by a single point separated from the cell's center by the magic distance. However, since we are dealing with rectangular cells it will be more symmetrical to use 4 observer points disposed along the rectangles' medians and all at the magic distance from the center.

In principle, this simple arrangement, reproduced in all the cells, will guarantee perfect results for the diagonal matrix terms and a moderate error in the near-diagonal terms. Moreover, this error should vanish as we go far away from the diagonal matrix elements correspond in principle to interactions between cells separated by larger distances.

More complex distributions of source and/or observer points (they can be exchanged by reciprocity) will provide additional degrees of freedom allowing the obtention of exact values in the Z-matrix not only for the diagonal terms but also for other off-diagonal terms. In this last case, the magic distance value is used as starting point for a fast MatLab-based optimization. As an example, using 2 groups of 4 observation points each, identified by the distances d1 (green) and $\mathrm{d} 2$ (red) in fig. 1, it is possible to enforce the matching condition on the self- and on the first near-terms.

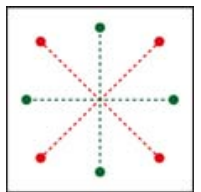

Figure 1. Configuration with 2 groups of 4 observation points.

In all the cases, the generic entry of the $\mathrm{Z}$ matrix is therefore calculated as:

$$
z_{i j}=\sum_{m=1}^{M} \frac{1}{\sqrt{\left(x_{i, m}-x_{j}\right)^{2}+\left(y_{i, m}-y_{j}\right)^{2}}}
$$


both for diagonal and non-diagonal matrix elements.

The above qualitative (and somewhat speculative) considerations are now confirmed with a practical IE application where we consider a unit square patch above an infinite ground plane.

In a first run, a full Galerkin approach was compared with the method using a single cross of four observation points all located at the magic distance (eqn. 11) from the cell's center. The use of the magic distance produces an error in the MoM matrix which is maximum on the first near-diagonal term and is always less than $6 \%$, as seen in fig. 2a. Once the MoM matrix is inverted the unknown charge surface charge density can be computed. Its values approximate very well the "exact" Galerkin solution (fig. 2b) and the error is on the capacitance is around $1.5 \%$.
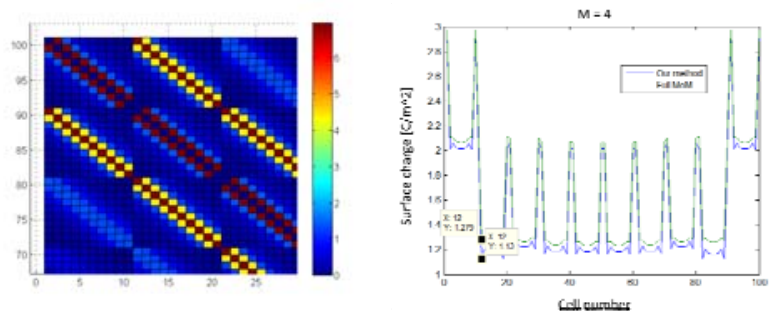

Figure 2. (a) Detail of the error on the MoM matrix. (b) Surface charge.

For a more accurate approximation, eight observation points located as depicted in fig. 1 are now used on each cell. The additional degree of freedom is used to minimize the error on the closest-to-diagonal terms (interactions between adjacent cells). The error in the MoM matrix elements is dramatically reduced (fig. 3a), the surface charge is very accurately reproduced (fig. 3b) and the error on the capacitance is reduced below $0.5 \%$.

The speed-up factor is obvious since using the magic distance strategy the number of elementary computations needed to evaluate the matrix elements is always the same (four or eight), regardless of the source-observer distance while Galerkin will require an complex treatment for cancelling the singularity of the the self-term and at least $M^{4}$ elementary computations The needed order of the quadrature rule, $M$ oscillates typically between 4 and 16 .
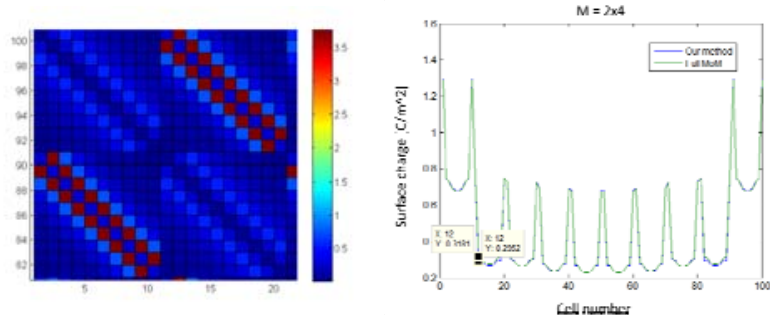

Figure 3. (a) Detail of the error on the MoM matrix. (b) Surface charge.

\section{THE ELECTRODYNAMIC FULL WAVE PROBLEM}

The full-wave problem presents some aspects that differ from the static problem and need to be treated. The Green's function is now the dyadic $\overline{\overline{\mathbf{G}}}_{\mathbf{E J}}$ (eqn. 1b), which strongly singular. In the integral equation (3) the unknown is the surface current $\mathbf{J}$ which is now a vector quantity. Moreover, using the standard MoM-Galerkin formulation the basis and test functions are defined by couples of adjacent cells rather than by individual cells.

This shows clearly that the determination of a single full-wave magic distance for self-terms is not possible, since in standard electrodynamic formulations the diagonal terms includes both the interaction of a cell with itself and with the adjacent one. Therefore, the simplest scheme is still inspired by the magic distance concept but involves at least an additional degree of freedom, like the configuration depicted in fig. 1.

Finally, the higher singularities in the electrodynamic case call for higher-order basis and test functions (typically the surface current density is expanded with linear rooftops rather than constant pulses). Therefore, to obtain a proper point-wise approximation of a Galerkin method, the different source and observation points must be associated to specific weights related to the basis and test functions being considered.

This last statement can be easily clarified considering the exact expression for the generic $z_{i j}$ term of the MoM matrix:

$z_{i j}=\int_{S_{i}} d s \mathbf{b}_{\mathbf{i}}(\mathbf{r}) \int_{S_{j}} d s^{\prime} \mathbf{b}_{\mathbf{j}}\left(\mathbf{r}^{\prime}\right) \overline{\overline{\mathbf{G}}}_{\mathbf{E J}}\left(\mathbf{r}, \mathbf{r}^{\prime}\right)$

If all the possible source-observer interactions are reduced to a single source point $\mathbf{r}_{\mathbf{j} 0}$ and $M$ observation points $\mathbf{r}_{\mathbf{i m}}$ then, the obvious approximation for eqn. 15 is:

$z_{i j}=\mathbf{M}_{\mathbf{j}} \sum_{m=1}^{M} \overline{\overline{\mathbf{G}}}_{\mathbf{E J}}\left(\mathbf{r}_{\mathbf{i m}}, \mathbf{r}_{\mathbf{j} \mathbf{0}}\right) \mathbf{M}_{\mathbf{i m}}$

where

$\mathbf{M}_{\mathbf{j}}=\int_{S_{j}} d s^{\prime} \mathbf{b}_{\mathbf{j}}\left(\mathbf{r}^{\prime}\right)$

is the total moment of the $\mathrm{j}$-th basis function and

$\mathbf{M}_{\mathbf{i m}}=\int_{\sigma_{i m}} d s^{\prime} \mathbf{b}_{\mathbf{j}}\left(\mathbf{r}^{\prime}\right)$

are the partial moments corresponding to the partial regions $\sigma_{i m}$ associated to the m-th observation point in the i-th cell.

Indeed, these partial moments can be considered as a vectorized form of the classical weights in numerical quadratures.

In order to numerically ascertain the validity and accuracy of these ideas a square metallic patch was considered. As in the static case, the patch was meshed with $10 \times 10$ square cells. The patch linear size is one free-space wavelength $\lambda$ and the excitation term in the EFIE was provided by a plane wave $(\mathbf{k}\|\mathbf{z}, \mathbf{E}\| \mathbf{x})$.

In the following developments, the optimum location of the testing points and the associated weights were obtained as the solution of a optimization problem using a well defined cost function and the MATLAB built-in optimization algorithm with the magic distance as initial guess. 


\section{Simple single source $1 \times(2 \times 4)$ algorithm}

The first logic attempt consists in applying a source/observation point scheme as in the electrostatic problem, i.e. $\mathrm{N}=1$ source points and $\mathrm{M}=2 \times 4$ observation points, as in fig. 1 . The most critical terms after the diagonal self-terms are those corresponding to overlapping basis and test functions that share one cell. Here, two different cases arise, depending on whether the basis/test functions are parallel or perpendicular. In order to deal with these two cases, an additional degree of freedom is needed. This is easily obtained if the cross' arms do not have necessarily the same length leaving to the modified configuration of fig. 4 .

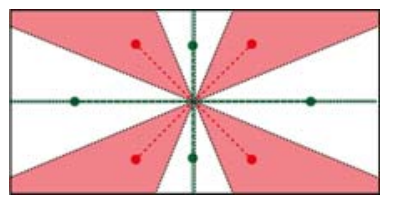

Figure 4. Configuration with 2 groups of 4 points each

The weights associated to the points can be easily obtained, according to (14), associating to every test point angular regions defined by the bisectors of the position vectors of the test points (fig. 4) with the results (for, respectively point in the green $x$-axis, red, green y-axis):

$$
\begin{aligned}
& \frac{L^{2}}{3} \tan \left(\frac{\pi}{8}\right) \\
& \frac{L^{2}}{4} \cot \left(\frac{3 \pi}{8}\right)-\frac{L^{2}}{24} \cot ^{2}\left(\frac{3 \pi}{8}\right) \\
& \frac{L^{2}}{4}-\frac{L^{2}}{6} \tan \left(\frac{\pi}{8}\right)+\frac{L^{2}}{48} \cot \left(\frac{3 \pi}{8}\right)-\frac{L^{2}}{8} \cot ^{2}\left(\frac{3 \pi}{8}\right)
\end{aligned}
$$

\section{Improved single source $1 x(3 \times 4)$ algorithm}

Following the previous described approach, it is straightforward to increase the number of observation points at will until reaching a prescribed accuracy.

Fig. 5 shows a very good compromise between complexity and accuracy, obtained with still a unique single source point and still only $3 \times 4$ observation points.

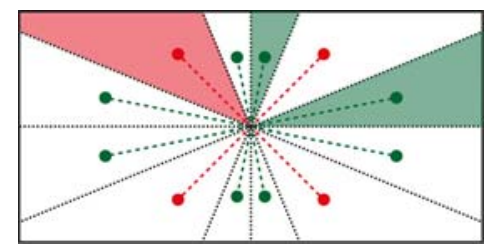

Figure 5. Configuration with 3 group of 4 points each

When tested on the one wavelength square plate, this point-wise scheme produced a satisfactory MoM matrix. Fig. 6(a) shows the error on the first row of this MoM matrix. The diagonal and near diagonal terms are exactly computed and the error in the most significative MoM matrix elements is below 3\% with an RMS error of $6.5 \%$. Such results make meaningful to invert the matrix to compute the surface current and compare it with the result of a standard MoM-Galerkin algorithm. Results are shown in Fig. 6(b).

The dominant component of the current is obviously the one parallel to the excitation field and correspond to basis (91-180) in fig. 6b. This component of the current is represented with satisfying accuracy: the current peaks are caught and only a small deviation is observed (RMS below 10\%). The stronger error which affects the quadrature (y-directed) is less relevant, taken into account the much lower values of this current-component. The RMS error of around $15 \%$ could be tolerated in most practical applications.
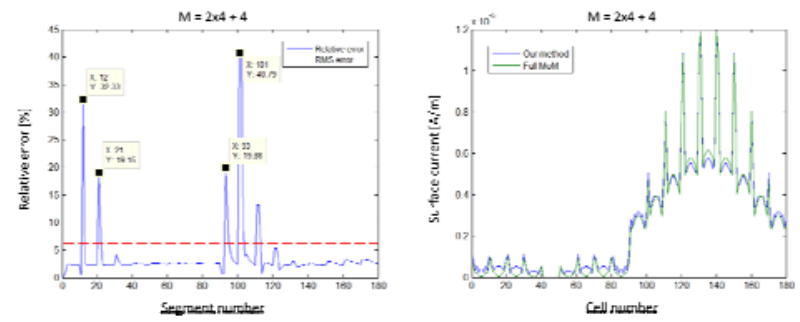

Figure 6. (a) Relative error on the first row of the MoM matrix and RMS error. (b) Surface current.

\section{Double source (2x6) algorithm}

The above described algorithms provide quite satisfactory results but they are unnecessarily unbalanced in terms of source and observation points. Indeed, according to reciprocity theorems, more satisfactory situations should be obtained by using closer numbers of source and observation points.

Extensive numerical trials have shown the amazing benefits obtained by just using $\mathrm{N}=2$ source points and keeping a magic distance inspired configuration in the observation points. A fast optimization led to the configuration of fig. 7 , using only $M=6$ observation points, thus halving the computational effort with respect to the previous configuration.

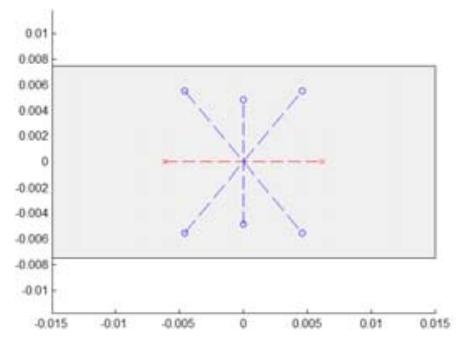

Figure 7. Configuration of $N=2$ source points and $M=2+4$ observation points.

The error in the MoM matrix entries is shown in Fig. 8(a), and it is remarkable that most elements show now an error below $2 \%$ with the 
RMS error quite small (5\%). The sporadic higher errors in some specific MoM matrix entries do not affect the overall quality of the results. This is clearly demonstrated in fig $8 \mathrm{~b}$ where the surface current density, obtained after matrix inversion, matches perfectly the MoM-Galerkin values. Indeed, Fig. 9 provides a qualitative comparison of the surface currents on the whole patch and confirms the excellence of this last algorithm.
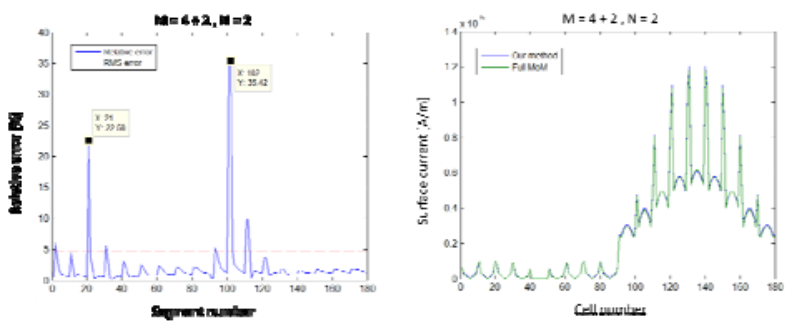

Figure 8. (a) Relative error on the first row of the MoM matrix and RMS error. (b) Surface current.
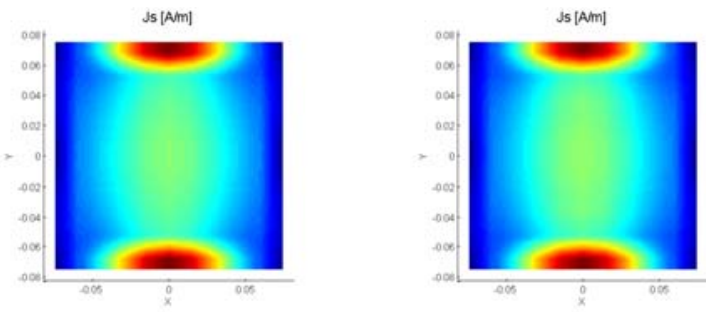

Figure 9. Surface currents on the patch. (a) Presented method and (b) Full MoM.

\section{CONCLUSIONS}

In this paper, a point-wise approach that can be used efficiently in the numerical solution of Electric Field Integral Equations has been presented. The algorithm is based on the so-called magic distance concept which defines exactly the point-to-point equivalent of a four dimensional integral. Magic distance values are rigorously obtained in the electrostatic case and generalized to the electrodynamic case via the Mixed Potential formulation of the Electric Field Integral Equation.

With the presented approach, point-matching schemas can now be also applied to the direct EFIE formulation without need of casting it in terms of auxiliary potentials. The advantages are manifold. On one side, it is possible to deal directly with field values, which are physical quantities always univocally defined. On the other hand, the procedure needed to obtain the MoM matrix is much simplified and the computational effort is greatly reduced when compared with a Galerkin formulation, all this without appreciable deterioration in the overall accuracy.

Work is in progress to extend these concepts to triangular meshes and more complex environments requiring alternate definitions of the involved Green's functions.
1. Balanis, C. A., Advanced Engineering Electromagnetics, John Wiley \& Sons, 1989

2. Michalski, K.A.; Mosig, J.R.; , "Multilayered media Green's functions in integral equation formulations," Antennas and Propagation, IEEE Transactions on , vol.45, no.3, pp.508-519, Mar 1997

3. Harrington RF. Field Computation by Moment Methods. Macmillan, Krieger: New York, FL, 1983.

4. Mosig J.R., Hall R.C., Gardiol F.E., Numerical analysis of microstrip patch antennas. In Handbook of Microstrip Antennas, James JR, Hall PS (eds). IEE-Peter Peregrinus: London, 1989.

5. Michalski, K.A., Zheng, D., "Electromagnetic scattering and radiation by surfaces of arbitrary shape in layered media. I. Theory," Antennas and Propagation, IEEE Transactions on , vol.38, no.3, pp.335-344, Mar 1990

6. Peterson, A.F., "Application of the locally corrected Nyström method to the EFIE for the linear dipole antenna," Antennas and Propagation, IEEE Transactions on, vol.52, no.2, pp. 603-605, Feb. 2004

7. Tong M. S., Chew W. C., "A higher-order Nyström scheme for electromagnetic scattering by arbitrarily shaped surfaces," Antennas and Wireless Propagation Letters, IEEE, vol.4, no., pp. 277- 280, 2005

8. Michalski, K.; , "On the scalar potential of a point charge associated with a time-harmonic dipole in a layered medium," Antennas and Propagation, IEEE Transactions on , vol.35, no.11, pp. 1299- 1301, Nov.1987

9. Polimeridis A. G. and J.R. Mosig, J.R., "Complete semi-analytical treatment of weakly singular integrals on planar triangles via the direct evaluation method", Int. J. Numerical Methods Eng., Vol. 83, pp. 16251650, 2010.

10. Durand, E., Électrostatique, Masson, 1964

\section{REFERENCES}




\section{BIOGRAPHIES}

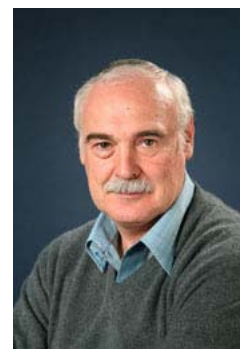

Juan R. Mosig was born in Cadiz, Spain. He received the Electrical Engineer degree from the Universidad Politecnica de Madrid, Madrid, Spain, in 1973, and the Ph.D. degree from the Ecole Polytechnique Fédérale de Lausanne (EPFL), Lausanne, Switzerland, in 1983. Since 1991,

he has been a Professor at EPFL and since 2000, he has been the Head of the Laboratory of Electromagnetics and Acoustics (LEMA) at EPFL.

In 1984, he was a Visiting Research Associate with the Rochester Institute of Technology, Rochester, NY, and Syracuse University, Syracuse, NY. He has also held scientific appointments with the University of Rennes, France, the University of Nice, France, the Technical University of Denmark at Lyngby and the University of Colorado at Boulder, USA.

$\mathrm{He}$ is a also a Fellow of the IEEE, and a founding member and acting Chair of the European Association\&Conferences on Antennas and Propagation (EurAAP and EuCAP).

Dr. Mosig has authored five chapters in books on planar antennas and circuits and over 100 peer-reviewed journal papers. His current research interests include electromagnetic theory, numerical methods, and planar antennas. 